\title{
KARLSRUHE
}

April 1971

KFK 1414

Institut für Neutronenphysik und Reaktortechnik

Siedeversuche an offenen Rillenkapillarverdampfern

F. E. Reiß, K. Schretzmann

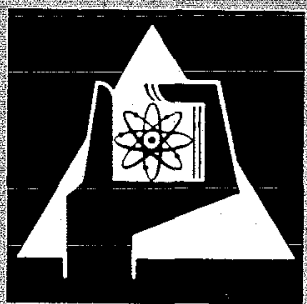

CESELLGHAFT FUR KERNFORSCHUNG M.B.H. 



\section{Siedeversuche an offenen Rillenkapillarverdampfern}

Von Friedrich Ernst Reiß und Klaus Schretzmann, Karlsruhe

Aus offenen Rillenkapillarverdampfern wurde Wasser verdamptt. Messungen lieferten den Wasser. verbrauch und die Verdampfertemperatur in Abhängigkeit von der zugeführten Heizleistung. Der ebenfalls gemessene Druckverlanf in den Kapillaren beim Austrocknen der Verdampter ließ sich mit Krï̈mmungsänderungen der Flüssigkeitsoberfläche erklären. Untersuchungen zum Spritzen der Verdampfer wurden ebenfalls vorgenommen.

\section{Versuchsanordnung}

Zum Untersuchen dër Wärmeübertragung und des Verdampfungsvorgangs bei Kapillarverdampferm dienten ebene Kapillarverdampfer mit Rillen, in denen man Wasser unter freier Atmosphäre verdampfte. Bild 1 zeigt die Bauart der verwendeten Verdampfer. Ein etwa $25 \mathrm{~mm} \times 50 \mathrm{~mm}$

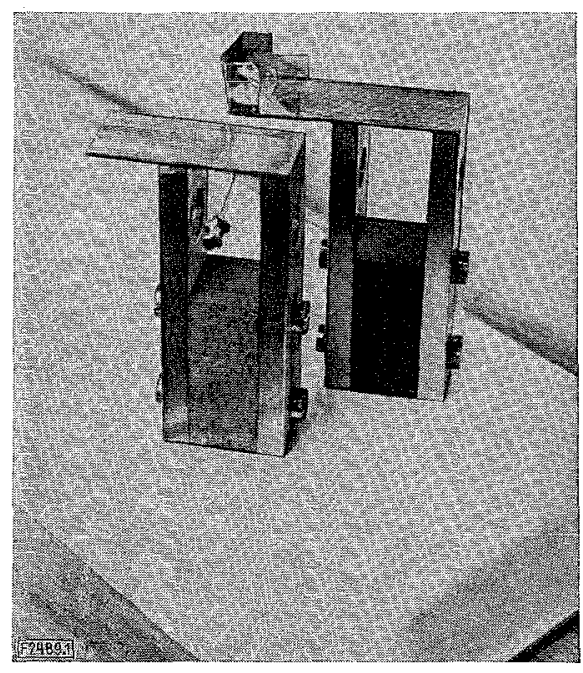

Bild 1. Rillenkapillarverdampfer.

großes, etwa 1,5 mm dickes Blech enthält durch Einsägen hergestellte, rd. $1 \mathrm{~mm}$ tiefe und untereinander parallele Rillen. Die Breite der Rillen und der sie trennenden Stege ist innerhalb bestimmter, von der Fertigungstechnik gesetzter Grenzen frei wählbar. Auf der Rückseite der Verdampferfläche setzen Stromzuführungsschienen verhältnismäßig großen Querschnitts an. Die beheizte Länge zwischen den Anschlüssen beträgt $20 \mathrm{~mm}$. Zwischen den Strom. zuführungen befindet sich ein eingeschraubter, elektrisch isolierender Klotz aus Pertinax, der die dünne Verdampferfläche mechanisch entlastet. Die Stromzuführungen sind so angeordnet, daß ein Teil der Kapillarfläche unbeheizt übersteht. Auf diesen Teil wird das zu verdampfende Wasser aufgegeben; mitunter ist zusätzlich eine aus Blech gebogene Aufgabemulde angebracht. Bie Verdampfer fertigte man einschließlich der Zuleitungen aus einem Stück; als Werkstoffe dienten bisher Messing Ms 58 und rostfreier Stahl WSt Nr. 1.4541.

Die Verdampfer wurden an die einwindige Sekundärwicklung eines $12-\mathrm{kW}$-Schnittbandkerns angeschlossen und durch direkten Stromdurchgang beheizt. Bild 2 zeigt den Versuchsaufbau. Die Heizströme von: einigen $1000 \mathrm{~A}$ wurden durch den Spannungsabfall an : einem wassergekühlten Meßwiderstand ermittelt. Die im Verdampfer umgesetzte Heizleistung berechnete man aus der gemessenen Heizstromstärke und dem aus den Abmessungen berechneten Wirkwiderstand des Verdampfers für. eine Werkstofftemperatur von $100^{\circ} \mathrm{C}$. 


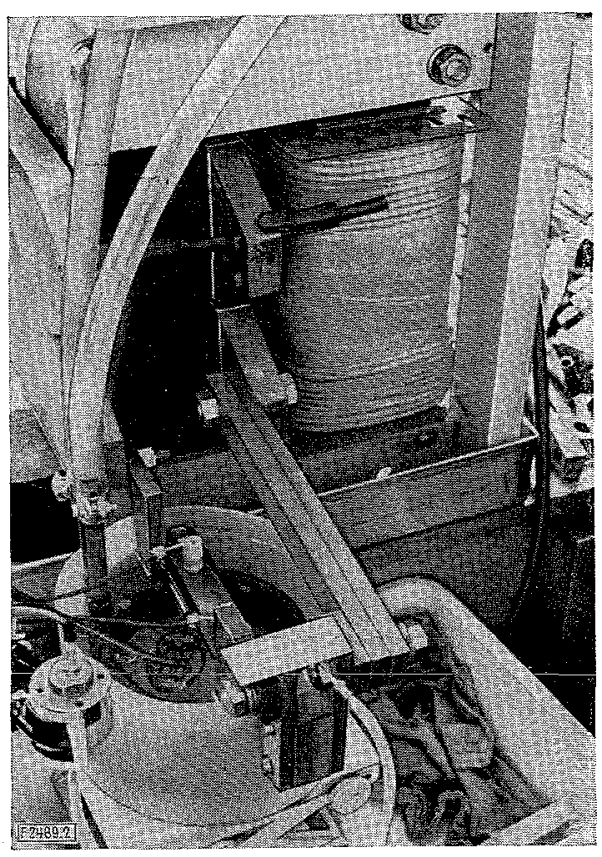

Bild 2. Versuchsaufbau mit betriebsfertig angeschlossenem Verdampfer.

\section{Wasserverbrauch}

An einer Anzahl von Verdampfern wurde ermittelt, wie der Wasserverbrauch im stationären Betrieb von der zugeführten Heizleistung abhängt. Bild 3 zeigt die gefundene Abhängigkeit. Sowohl die auf der Abszisse aufgetragene, dem Verdampfer zugeführte Bruttoheizleistungsdichte $\dot{q}$ als auch der auf der Ordinate aufgetragene spezifische Wasserverbrauch $\dot{m}$ sind auf die Gesamtfläche des Verdampfers bezogen. Die in das Bild eingezeichnete Gerade I stellt den Idealzustand dar, daß gerade soviel Wasser verdampft, wie der zugeführten elektrischen Heizleistungsdichte entspricht. Kurve II gleicht die Meßpunkte für die Messing-Verdampfer aus.

Bei kleinen Leistungsdichter ist $\dot{m}$ infolge der Verluste durch Wärmeableitung in die Anschlüsse und durch Konvektion erheblich kleiner als der zugeführten Heizleistung entspricht. Bei Verdampfern aus rostfreiem Stahl sind die Verluste durch Wärmeableitung erwartungsgemäß deutlich kleiner als bei Messing-Verdampfern, wie die eingetragenen Meßpunkte zeigen. Bei großen Heizleistungsdichten liegt der Wasserverbrauch des Verdampfers merklich höher als der zugeführten Heizleistung entspricht, weil das schon bei verhältnismäßig kleinen Leistungsdichten einsetzende und immer weiter zunehmende Spritzen des Verdampfers einen erheblichen Teil des erst etwas angewärmten Wassers abführt. Die obere Schranke für den zur Dampferzeugung verwendeten spezifischen Wasserstrom ist durch die eingezeichnete Idealgerade I gegeben. Bei der höchsten, hier erreichten Wärmeleistungsdichte von $212 \mathrm{~W} / \mathrm{cm}^{2}$ verspritzt der Verdampfer etwa drei- bis viermal soviel Wasser wie er verdampft. Diese Erscheinung ist qualitativ durch visuelle Beobachtungen an mit Wasser betriebenen Wärme. rohr-Verdampfern [1] und an Quecksilber-Wärmerohren [2] bekannt.

Bei Leistungsdichten, die etwas unter den für den Einsatz des Spritzens nötigen Werten liegen, erscheinen Blasen, die beim Aufsteigen aus den Rillen Flüssigkeit aus diesen heraus auf die Stege drücken. Der Verdampfer ist dann auf seiner gesamten Oberfläche benetzt und verdampft ohne Rücksicht auf die Einzelheiten der Kapillarstruktur. Dies gilt bis zu den höchsten, bei den beschriebenen Versuchen erreichten Leistungsdichten, wie Bild 3 zeigt, und rechtfertigt bei diesem Betriebszustand das Berechnen der
Heizleistungsdichte mit der Gesamtoberfläche des Verdampfers. Der Einfluß der Kapillarstruktur, soweit sie für die Nachförderung von Flüssigkeit nötig ist, wurde durch eine waagrechte Anordnung des Verdampfers und insgesamt kurze Förderwege weitgehend ausgeschaltet.

\section{Verdampfertemperatur und Siedekennlinie}

Bei kleinen $\dot{q}$-Werten steigen die Temperaturen des Wassers und des Verdampfers noch deutlich mit der Heizleistungsdichte an. Bild 4 zeigt dies für einen Verdampfer aus rostfreiem Stahl. Dabei wurden die Wassertemperatur mit einem feinen Thermoelement in einer Rille etwa im Mittelpunkt der Verdampferfläche und die Heizertemperatur auf der Unterseite der Verdampferfläche (etwa in der Mitte) durch ein aufgepunktetes Thermoelement gemessen.

Wenn sich Sieden des Wassers einstellt, kann bei einer -Leistungserhöhung die Mehrleistung auf Grund einer verhältnismäßig kleinen Temperaturerhöhung des Verdampfers an das Wasser abgegeben werden. Die Wärmeableitung zu den auf der Kühlwassertemperatur gehaltenen Anschlüssen des Verdampfers erhöht sich dadurch nur wenig.

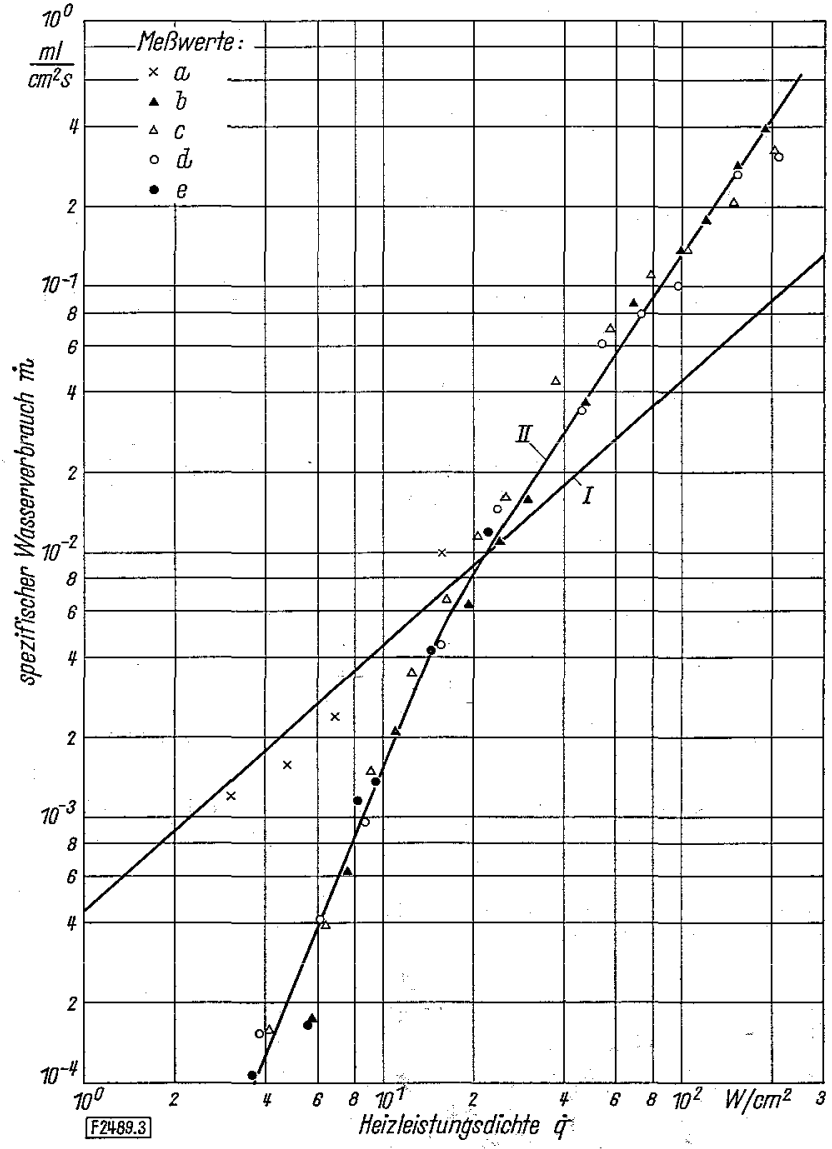

Bild 3. Spezifischer Wasserverbrauch $\dot{m}$ von Rillenver. dampfern in Abhängigkeit von der Heizleistungsdichte $\dot{q}$.

I Idealgerade, II Ausgleichskurve dưrch die MeBpunkte für Messing Verdampfer; Bedeutung der Meßpunkte:

\begin{tabular}{|c|c|c|c|c|}
\hline \multirow{2}{*}{$\begin{array}{c}\text { Bezeich- } \\
\text { nung } \\
-\end{array}$} & \multirow{2}{*}{$\begin{array}{c}\text { Werkstoff } \\
-\end{array}$} & \multicolumn{3}{|c|}{ Abmessung } \\
\hline & & $\begin{array}{c}\text { Rillenbreite } \\
\text { mm }\end{array}$ & $\begin{array}{l}\text { Rillentiefe } \\
\mathrm{mm}\end{array}$ & $\begin{array}{c}\text { Stegbreite } \\
\mathrm{mm}\end{array}$ \\
\hline $\begin{array}{l}a \\
b \\
c \\
d \\
e\end{array}$ & $\begin{array}{c}\text { Stahl } \\
\text { Messing } \\
\text { ", } \\
\text { " } \\
\text { "" }\end{array}$ & $\begin{array}{l}0,8 \\
0,28 \\
0,28 \\
0,28 \\
0,28\end{array}$ & $\begin{array}{l}0,95 \\
0,90 \\
0,90 \\
0,90 \\
0,90\end{array}$ & $\begin{array}{l}0,5 \\
0,22 \\
0,5 \\
1,0 \\
2,0\end{array}$ \\
\hline
\end{tabular}




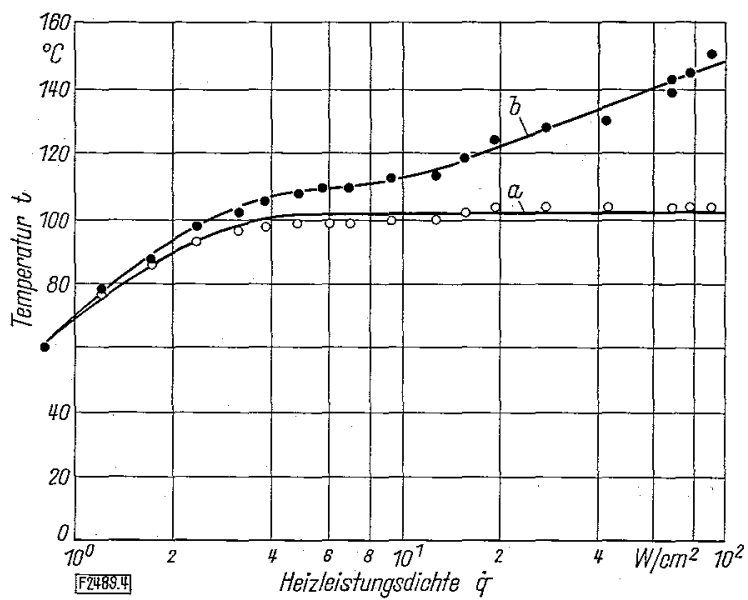

Bild 4. Temperatur $t$ an einem Heizer in Abhängigkeit von der Fieizleistungsdichte $\dot{q}$.

Werkstoff: rostfreier Stahl; Rillenbreite $0,8 \mathrm{~mm}$, Rillentiefe $0,95 \mathrm{~mm}$, Stegbreite $0,5 \mathrm{~mm}$; a Wasser, b Heizerrückseite

Bei hohen Leistungsdichten $\left(>25 \mathrm{~W} / \mathrm{cm}^{2}\right)$ fällt der Anteil der Wärmeableitung in der Auftragung von Bild 3 nicht mehr ins Gewicht.

Aus dem Wärmefluß an das Wasser und der in Bild 4 gemessenen Temperatur der Heizerunterseite kann man die Temperaturdifferenz quer durch den Heizerwerkstoff abschätzen sowie hiermit aus den gemessenen Temperaturen der Heizerunterseite die Temperatur der dem Wasser zugewendeten Oberfläche ausrechnen. Zusammen mit den ebenfalls in Abhängigkeit von der Heizleistungsdichte gemessenen Wassertemperaturen läßt sich aus diesen Daten

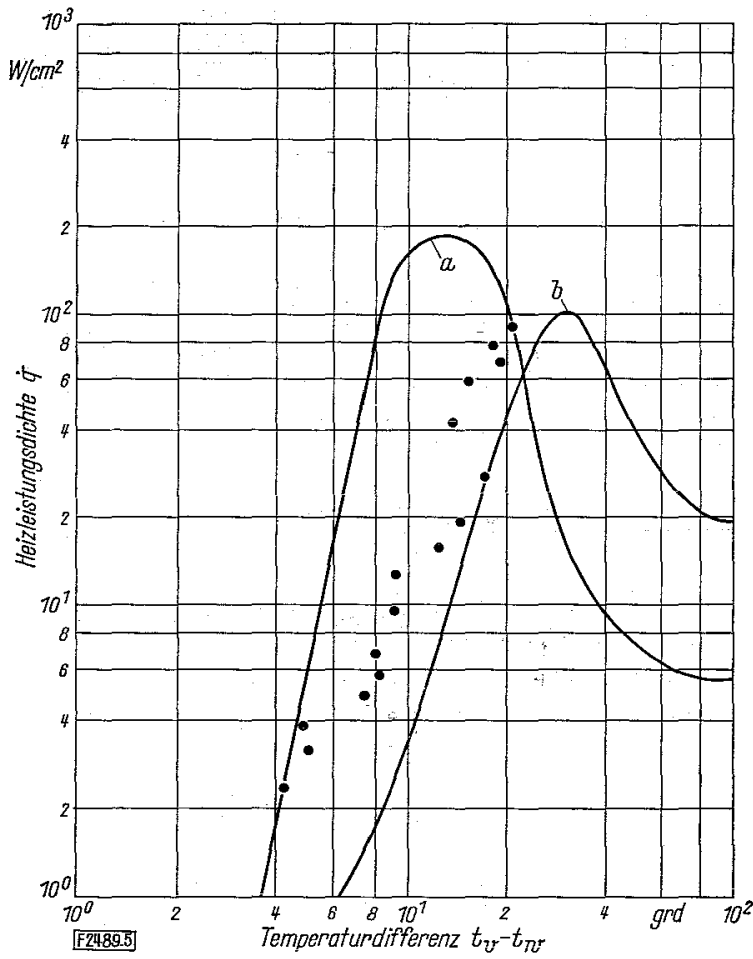

Bild 5. Siedekennlinie eines Rillenverdampfers.

Verdampferflüssigkeit Wasser unter Atmosphärendruck, Verdampferwerkstoff und Rillenabmessungen wie bei Bild 4 ; $t_{\mathrm{v}}$ und $t_{\mathrm{w}}$ Temperatur der Verdampferoberfläche bzw. des Wassers, a Siedelinie nach $[3 ; 4]$ b Siedelinie nach [5]; die Punkte entsprechen den MeBwerten. ein Teil der Siedekennlinie des Kapillarverdampfers aufzeichnen, Bild 5. In doppeltlogarithmischem Maßstab wurde hier über der Differenz der Temperaturen von Wand und Wasser die an das Wasser übertragene Leistungsdichte $\dot{q}$ für einen Verdampfer nach Bild 1 aus rostfreiem Stahl mit 18 Rillen aufgetragen. Die höchste Leistungsdichte (bezogen auf die Gesamtoberfläche) betrug hier $91 \mathrm{~W} / \mathrm{cm}^{2}$. Der bei diesem Versuch verwendete Heiztransformator ließ keine höhere Leistung zu. Die im Schrifttum angegebenen Siedekennlinien von Verdampfern mit glatten Oberflächen beim Behältersieden weichen sehr voneinander ab. In Bild 5 wurden eine aus den Wärmeübergangswerten nach $[3 ; 4]$ berechnete Siedekennlinie $a$ und die Siedekennlinie $b$ nach [5] eingezeichnet. Die Meßergebnisse liegen innerhalb des Schwankungsbereichs der beiden Linien $a$ und $b$.

Die auf der Abszisse in Bild 5 aufgetragenen Temperatur. differenzen für die Meßpunkte sind mit einer hohen Unsicherheit behaftet, weil die Fühlstelle des für die Tem: peraturmessung auf der Heizerunterseite benutztenThermoelements etwa so breit wie die Rille des Verdampfers war. Damit bleibt unklar, zu welchem Punkt des Temperaturfeldes der Heizerunterseite die gemessene Temperatur gehört bzw. welchen Abstand zur wasserbenetzten Oberfläche des Heizers man bei der Berechnung der Temperaturdifferenz im Heizerwerkstoff benutzen soll.

\section{Druckverlauf}

Die Untersuchungen erstreckten sich weiter auf den Druckverlauf in der Flüssigkeit beim Austrocknen des Verdampfers. Dazu wurde mit einemQuarzdruckaufnehmer der Druck am Rillengrund gemessen. Zur Druckentnahme diente eine im Rillengrund eingelötete Injektionsspritzenkanüle (vgl. Bild 1). Zwecks Vermeidens von zusätzlichen Menisken, deren Krümmungsänderung während der Mossung das Ergebnis verfälschen würde, hatte man das gesamte Meßsystem (Quarzdruckaufnehmer, Schlauch, Kanüle) mit einer zusammenhängenden Wassersäule ge-

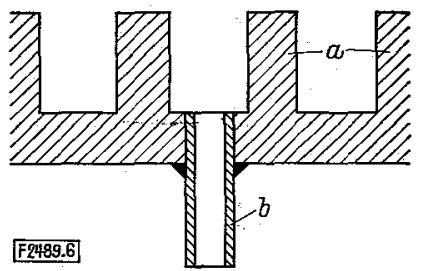

Bild 6. Schema der Druckentnahmestelle am Rillenverdampfer.

a Stege, b Kanüle

füllt. Bild 6 zeigt das Schema der Druckentnahmestelle. Die Zeitkonstante des nur quasistatische Messungen zulassenden Quarzsystems (einschließlich des Verstärkers) lag um mindestens eine Größenordnung über der Dauer einer „Austrocknung ", die zwischen 20 und 200 s betrug.

Bild 7 zeigt den grundsätzlichen Verlauf der erhaltenen Druckkurven. Als Nullpunkt für den Druck gelte der Zustand des mit ebener Oberfläche gefüllten Verdampfers bei Punkt a. Verdampft man, ohne Wasser nachzufüllen, so wird sich die Wasseroberfläche in die Rille zurückziehen und krümmen. Der Druck in der Flüssigkeit sinkt. Dieser Unterdruck ist es, der im Wärmerohr Flüssigkeit in den Verdampfer nachsaugt. Der Druck fällt bis zum Punkt $b$ mehr oder weniger regelmäßig um einen von der Rillenbreite abhängigen Betrag ab. Eine visuelle Beobachtung der Verdampfer zeigt, daß in diesem Zustand die Flüssigkeit in der Rille bis zum Rillenboden abgesunken ist. Einen Augenblick später reißt der den Rillenboden noch benetzende Flüssigkeitsfilm auf. Der Rillenboden wird 


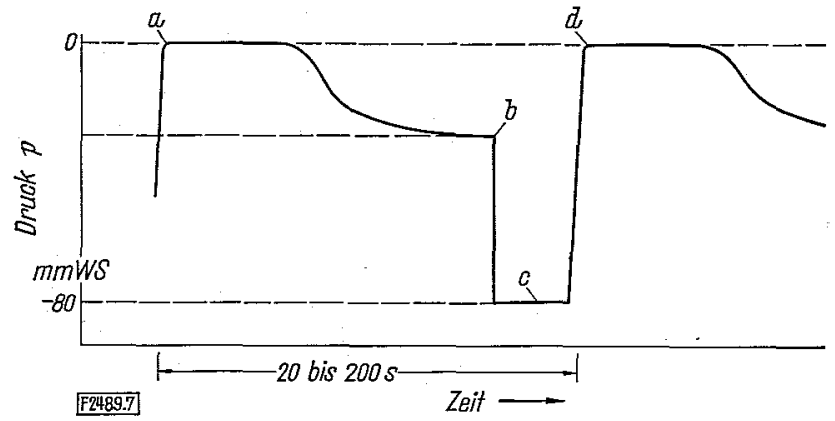

Bild 7. Druckverlauf beim Trockenfahren wassergefüllter Rillenkapillaren.

a bis d im Text erläuterte Stellen; Gegenüberstellung der gemessenen und der berechneten Drücke:

\begin{tabular}{c|c|c|c|c}
\hline \multirow{2}{*}{$\begin{array}{c}\text { Rillenbreite } \\
\text { mm }\end{array}$} & \multicolumn{2}{|c|}{ Kapillardruck der Rille } & \multicolumn{2}{|c}{$\begin{array}{c}\text { Kapillardruck der Bohrung } \\
(0,3 \mathrm{~mm} \text { Dmr. }\end{array}$} \\
\hline mm WS & $\begin{array}{c}\text { berechnet } \\
\text { nm WS }\end{array}$ & $\begin{array}{c}\text { gemessen } \\
\text { mm WS }\end{array}$ & $\begin{array}{c}\text { berechnet } \\
\text { mm WS }\end{array}$ \\
\hline 1,0 & 13 & 12 & 76 & 80 \\
0,5 & 30 & 24 & 82 & 80 \\
0,28 & 46 & 48 & 75 & 80 \\
\hline
\end{tabular}

trocken; der Meniskus springt in die Druckentnahmebohrung zurück, Punkt c. Um ein Überhitzen des nun trockenen Verdampfers zu vermeiden, wird er wieder bis zum Herstellen der ebenen Oberfläché geflutet, Punkt d.

Aus Bild 7 kann man die gemessenen Druckunterschiede vom vollen Verdampfer bis zur gerade noch benetzten Rille sowie den Drucksprung für den Rückzug des Meniskus in die Druckentnahmebohrung für drei verschiedene Rillenbreiten im Vergleich mit berechneten Werten ersehen. Dabei wurde für die Rechnung vollständige Benetzung und für die Rille eine halbzylindrische, für die Bohrung eine halbkugelige Form des Meniskus angenommen. Für die Oberflächenspannung des Wassers legte man den Wert bei $100{ }^{\circ} \mathrm{C}$ zugrunde. Die Übereinstimmung zwischen gemessenen und berechneten Werten ist brauchbar.

Die durch Krümmungsänderungen hervorgerufenen Drucksprünge überwiegen bei weitem alle anderen Effekte. So betrugen die Änderung dēs hydrostatischen Drucks beim Austrocknen der Rille rd. $1 \mathrm{~mm}$ WS, die dynamische Druckerniedrigung in der Strömung zwischen $10^{-4}$ und $10^{-5} \mathrm{~mm}$ WS sowie dex Druckabfall in Strömungsrichtung (laminarer Fall, $1 \leqq$ Re $\leqq 5$ mit Re als der Reynoldszahl) etwa $0,3 \mathrm{~mm} W \mathrm{~W} / \mathrm{cm}$ bei $2 \mathrm{~cm}$ insgesamt beheizter Länge.

\section{Einsetzen des Spritzens}

Es wurde versucht, ein Kriterium für das Einsetzen des Spritzens zu gewinnen. Der Spritzbeginn hängt nicht in einfacher Weise mit der Leistungsdichte und der Rillenbreite zusammen. Man kann bei gleichen sonstigen Bedingungen den Spritzbeginn zu höheren Leistungsdichten hin verschieben, wenn man die Durchgänge verengt, durch die der Dampf entweicht. Es wurden in die Rillen verschiedener Verdampfer Blechstreifen oder Runddrähte eingelegt (und dabei sichergestellt, da.B in diesen Einlagen keine zusätzliche Leistungserzeugung auftrat). Durch diese Verengung der für den Dampfaustritt wichtigen Spalte auf $\mathrm{rd} .1 / 5$ der
Breite der gesamten Rille stieg die zum Spritzen nötige Leistung um Faktoren zwischen 2 und 4. An Verdampfern, die sich nur in der Rillenbreite unterscheiden, steigt die zum. Spritzen nötige Leistungsdichte mit abnehmender Rillenbreite, z. B. von $2 \mathrm{~W} / \mathrm{cm}^{2}$ bei $1 \mathrm{~mm}$ Rillenbreite auf etwa $10 \mathrm{~W} / \mathrm{cm}^{2}$ bei $0,28 \mathrm{~mm}$ Rillenbreite. Die Rillentiefe beträgt dabei einheitlich etwa $1 \mathrm{~mm}$. Dagegen spritzt eine ebene beheizte Fläche, die von 1 bis $2 \mathrm{~mm}$ Wasser überdeckt wird, bei Leistungsdichten um $15 \mathrm{~W} / \mathrm{cm}^{2}$. Die Tropfen im Sprühnebel werden mit abnehmender Breite der Dampfpassagen kleiner.

Ein Kriterium, ob die Trägheitskräfte der durch die Dampfblasen gerührten Flüssigkeit die Oberflächenkräfte überwiegen, ob sich also Tropfen ablösen können, gibt nach [6] die Weberzahl We $=\varrho v^{2} l / \sigma$ mit $\varrho$ als der Flüssigkeitsdichte, $\sigma$ als der Oberflächenspannung, $v$ als einer charakteristischen Geschwindigkeit und $l$ als einer charakteristischen Länge der Siedeanordnung.

Man kann von der Vorstellung ausgehen, daß die sich am Rillengrund bildenden Blasen der Flüssigkeitsoberfläche entgegenwachsen. Wenn der obere Rand der Blase die Flüssigkeitsoberfläche durchbricht, zerreißt der Flüssigkeitsfilm; von den zurückweichenden Flüssigkeitsrändern werden die Spritztropien abgerissen oder abgeschleudert. Die Grenze für den Beginn des Spritzens $(\mathrm{We}=1)$ wurde für mehrere Rillenbreiten unter der Annahme abgeschätzt, daß $v$ die Geschwindigkeit der oberen Grenzfläche der in der Rille wachsenden und aufsteigenden Blase und $l$ die Rillenbreite sei. Für Rillenbreiten von $1 \mathrm{~mm}$ ergibt sich die nötige Geschwindigkeit zu $v=24 \mathrm{~cm} / \mathrm{s}$ oder bei einem Aufstieg von $1 \mathrm{~mm}$ in der Rille eine Lebensdauer der Blase von etwa $5 \mathrm{~ms}$; für einen Verdampfer mit $0,28 \mathrm{~mm}$ breiter und $1 \mathrm{~mm}$ tiefer Rille erhält man analog ein Zeitintervall von rd. $18 \mathrm{~ms}$ bei Spritzbeginn.

Es wurde die Feinstruktur des Drucksignals (Bild 7) untersucht. Allerdings konnte man infolge des $50-\mathrm{Hz}$ Brumms der Verdampferheizung die volle Empfindlichkeit nicht ausnutzen. Wenn die Verdampfer spritzen, läßt sich noch eben ein Signal mit einer Zeitdauer von etwa $10 \mathrm{~ms}$ erkennen (einheitlich bei Verdampfern von $1,0,5$ und $0,28 \mathrm{~mm}$ Rillenbreite und unabhängig von der um einen Faktor 4 variierten Gesamtleistung). Die Untersuchungen hierzu laufen weiter.

\section{Schrifttum}

[1] Bähr, A., E. Burck u. W. Hufschmidt: Liquid-vapor interaction and evaporation in heat-pipes. Second Internat. Conf. on Thermionic Electrical Power Generation, Stresa 1968. Ispra: Euratom 1968.

[2] Deverall, J.E.: Mercury as a heat pipe fluid. Los Alamos Scientific Lab. Univ. of Calif., Los Alamos, New Mexico. LA-4300, Okt. 1969.

[3] Gröber, H., S. Erk u. U.Grigull: Die Grundgesetze der Wärmeübertragung. 3. Aufl. Berlin, Heidelberg, New York: Springer-Verlag 1961.

[4] Farber, E. A., u. R. L. Scorah: Heat transfer to water boiling under pressure. 'Irans. Amer. Soc. Mech. Engrs. (ASME) 70 (1948) Nr. 5 S. 369/84.

[5] VDI-Wärmeatlas. Düsseldorf: VDI-Verlag 1963.

[6] Streeter, V.L.: Fluid mechanics. 4. Aufl. New York, Toronto, London, Sydney: McGraw-Hill Book Comp. 1966.

Eingegangen am 28.10.1970 